\title{
Risk of Major Hemorrhage after Kidney Transplantation
}

\author{
Manish M. Sood ${ }^{a, b} \quad$ Amit X. Garg ${ }^{c-e}$ Sarah E. Botac,d Limesh Marisiddappa ${ }^{a}$ \\ Eric McArthurc Kyla L. Naylor ${ }^{d}$ Moira K. Kapralc, f,g S. Joseph Kim c, f, h \\ Ngan N. Lam ${ }^{d, e}$ Amber O. Molnar ${ }^{a, b}$ Ziv Harel ${ }^{c, f, i}$ Jeffrey Perl ${ }^{f, i}$ Greg A. Knolla,b \\ ${ }^{a}$ Division of Nephrology, University of Ottawa and ${ }^{b}$ Clinical Epidemiology Program, Ottawa Hospital Research Institute, \\ Ottawa, Ont., ' Institute for Clinical Evaluative Sciences, ${ }^{\mathrm{d}}$ Department of Epidemiology and Biostatistics, Western

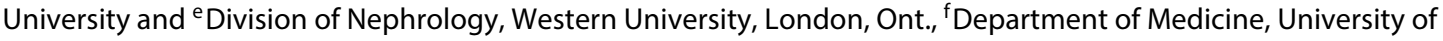 \\ Toronto, 9 The Institute of Health Policy Management and Evaluation, University of Toronto, h University Health Network, \\ Toronto General Hospital, and 'St. Michael's Hospital, Toronto, Ont., Canada
}

\section{Key Words}

Hemorrhage - Kidney transplantation - Gastrointestinal .

Subarachnoid · Epidemiology · Cumulative incidence .

Propensity score matched · Bleeding

\begin{abstract}
Background: Major hemorrhagic events are associated with significant morbidity and mortality. We examined the threeyear cumulative incidence of hospitalization with major nontraumatic hemorrhage after kidney transplantation. Methods: We performed a retrospective cohort study using healthcare administrative data of all adult-incident kidneyonly transplantation recipients in Ontario, Canada from 1994 to 2009. We calculated the three-year cumulative incidence, event rate, and incident rate ratio of hospitalization with major hemorrhage, its subtypes and those undergoing a hemorrhage-related procedure. Results were stratified by patient age and donor type and compared to a random and propensity-score matched sample from the general population. $\boldsymbol{R e}$ sults: Among 4,958 kidney transplant recipients, the threeyear cumulative incidence of hospitalization with nontraumatic major hemorrhage was $3.5 \%$ (95\% confidence interval [Cl] 3.0-4.1\%, 12.7 events per 1,000 patient-years) compared to $0.4 \%(95 \% \mathrm{Cl} 0.4-0.5 \%)$ in the general population ( $\mathrm{RR}=$
\end{abstract}

8.2, 95\% Cl 6.9-9.7). The crude risk of hemorrhage was 3-9fold higher in all subtypes (upper/lower gastrointestinal, intra-cranial) and 15-fold higher for gastrointestinal endoscopic procedures compared to the random sample from the general population. After propensity score matching, the relative risk for major hemorrhage and its subtypes attenuated but remained elevated. The cumulative incidence of hemorrhage was higher for older individuals and those with a deceased donor kidney. Conclusion: Kidney transplantation recipients have a higher risk of hospitalization with hemorrhage compared to the general population, with about 1 in 30 recipients experiencing a major hemorrhage in the three years following transplant.

(c) 2015 S. Karger AG, Basel

\section{Introduction}

As the prevalent kidney transplantation population grows, there is an increasing need to quantify the risk of general medical conditions to inform patients and to design appropriate interventions to minimize risk. Nontraumatic hemorrhagic events are a common cause of hospitalization in the general population and contribute significantly to patient morbidity, mortality and healthcare

\section{KARGER 125}

(c) 2015 S. Karger AG, Base

0250-8095/15/0411-0073\$39.50/0 
costs [1-5]. In kidney transplant recipients, the incidence of hemorrhage remains unknown. Previous studies have only been case reports or case series, limited to surgical or medication-related hemorrhage, with a relatively short duration of follow-up [6-12]. It is also unknown whether factors associated with hemorrhage in the general population, such as advancing age, or factors associated with increased complications in transplant recipients, such as donor kidney type, are associated with increased risk [13].

The goal of this study was to determine the three-year cumulative incidence of hospitalization with major nontraumatic hemorrhage and related gastrointestinal endoscopic procedures post-kidney transplantation and to stratify the risk of hemorrhage by age, source of organ (deceased or living), and timing (before or after 90 days of transplantation). To place the results into context, kidney transplant patients were compared to a random sample from the general population and sample matched by propensity scores.

\section{Methods}

\section{Design and Setting}

All adults ( $\geq 18$ years) who received a first-time kidney-only transplant in Ontario, Canada between January 1, 1994 and December 31, 2009, were identified by linked healthcare databases in Ontario, Canada (population 13 million) [14]. All healthcare encounters are prospectively entered in administrative databases and are held in a linked, de-identified form, and analyzed at the Institute for Clinical Evaluative Sciences (ICES). We conducted this study according to a pre-specified protocol that was approved by the research ethics board at Sunnybrook Health Sciences Centre (Toronto, Canada). The reporting of this study followed guidelines for observational studies (online suppl. table S1; for all online suppl. material, see www.karger.com/doi/10.1159/000371902) [15].

\section{Data Sources}

Patient characteristics, covariate information, and outcome data were obtained using records from four linked databases. We identified all patients who underwent kidney transplantation in Ontario through the Canadian Organ Replacement Registry (CORR) [16]. We obtained vital statistics from the Ontario Registered Persons Database (RPDB) that contains demographic information on all Ontario residents who have ever been issued a health card. We identified diagnostic and procedural information on all hospitalizations from the Canadian Institute for Health Information Discharge Abstract Database (CIHI-DAD). We obtained information from the Ontario Health Insurance Plan (OHIP) database, which includes health claims for inpatient and outpatient physician services. We have used a similar approach in previous publications regarding renal health outcomes, kidney transplant outcomes, and health services [17-22]. Whenever possible, we defined patient characteristics and outcomes using database codes that have been proven reliable when compared with manual chart review (online suppl. table S2).

\section{Population}

We included adult patients who received a first-time kidneyonly transplant within a 16 -year accrual period. The date of transplant served as the index date (also referred to as the cohort entry date). Patients were excluded if they were $<18$ years of age, received a previous transplant or a combined organ transplant such as a simultaneous pancreas kidney transplant (patient selection presented in online suppl. fig. S1). For comparison, we used a random sample of controls from the adult, non-transplant general population and matched on age, sex, and propensity score. The index date of the general population control group was a randomly chosen date within the accrual period. Individuals in the general population cohort had similar exclusion criteria as the transplant cohort with the addition of exclusion for a history of kidney disease.

Known demographics and comorbid risk factors for hemorrhage were captured in the three years prior to the index date, including hypertension, prior stroke, prior major hemorrhage, diabetes, and cardiovascular disease [23]. Variables associated with end-stage renal disease, dialysis modality, and the donor type were also captured. Delayed graft function was defined as having at least 1 code for dialysis in the first 7 days after transplantation.

\section{Outcomes}

The primary outcome was hospitalization with nontraumatic major hemorrhage; defined as an International Classification for Disease (ICD) code for hemorrhage from the upper and lower gastrointestinal tract, subarachnoid, intra-cerebral, or other hemorrhage at the time of hospitalization. Other hemorrhage was defined as other nontraumatic intracranial hemorrhage. The secondary outcome was the use of in-hospital gastrointestinal-related endoscopic procedures. All transplant recipients and general population controls were followed for three years for the occurrence of the primary or secondary outcome, death, or the end of the follow-up period. A three-year observation period was selected for two reasons: (i) a period of follow-up that could be reasonably adopted in a future interventional trial, testing interventions to prevent hemorrhage and (ii) aligns with any results from the United States as recipients have coverage and data collection for 3 years post-transplantation. $\mathrm{Pa}$ tients were not censored at the time of graft failure since this may lead to bias, given the possibility that loss of transplant function may be associated with bleeding. Hospitalizations for major hemorrhage were identified using ICD revisions 9 and 10 codes, in CIHI-DAD with validation in previous studies (online suppl. table S3) [24-26].

\section{Statistical Analysis}

Baseline characteristics were compared using standardized differences with a value greater than $10 \%$ considered significant. We calculated the three-year cumulative incidence of hospitalization with nontraumatic major hemorrhage (defined as the proportion of patients who experienced the event at least once within the three-years of follow-up) and the three-year incidence rate (defined as the rate per 1,000 person-years of follow-up). For the incidence rates, individuals were censored at the time of a specific type of hemorrhage but were eligible to contribute to subsequent different types of hemorrhage until death or the end of the followup period. However, individuals were eligible only to contribute once to the total major hemorrhage outcome. Cumulative incidence $95 \%$ confidence intervals were calculated using the Wilson score method. Cumulative incidences of the primary and secondary outcomes were compared to both the random and matched 
Table 1. Baseline characteristics of kidney transplant recipients compared to the general population

\begin{tabular}{|c|c|c|c|}
\hline Characteristics & Transplant recipients & General population* & Standardized differences** (\%) \\
\hline $\mathrm{n}$ & 4,958 & 100,000 & \\
\hline Age, mean \pm SD & $48.5 \pm 13.5$ & $43.3 \pm 16.3$ & 34.7 \\
\hline$\leq 44$ & 37.7 & 56.7 & 31.8 \\
\hline $45-64$ & 50.0 & 30.5 & 32.7 \\
\hline$\geq 65$ & 12.3 & 12.8 & 1.2 \\
\hline \multicolumn{4}{|l|}{ Income quintile, $\%$} \\
\hline 1 (lowest) & 22.0 & 21.0 & 2.0 \\
\hline 2 & 20.3 & 20.2 & 0.2 \\
\hline 3 (middle) & 20.0 & 19.7 & 0.6 \\
\hline 4 & 18.7 & 19.3 & 1.3 \\
\hline 5 (highest) & 18.8 & 19.5 & 1.5 \\
\hline Coronary artery disease & 30.6 & 4.0 & 55.3 \\
\hline Coronary revascularization ${ }^{\dagger}$ & 4.6 & 0.44 & 19.4 \\
\hline Atrial fibrillation & 2.1 & 0.4 & 11.3 \\
\hline Deep vein thrombosis & 1.5 & 0.1 & 11.3 \\
\hline Pulmonary embolism & 0.5 & 0.1 & 5.4 \\
\hline Diabetes mellitus & 25.7 & 6.2 & 41.6 \\
\hline Hypertension & 74.1 & 16.0 & 114.1 \\
\hline Peripheral artery disease & 11.9 & 0.20 & 36.0 \\
\hline Prior gastrointestinal endoscopy & 23.1 & 7.0 & 35.1 \\
\hline
\end{tabular}

* Random sample of the general population. ${ }^{* *}$ Standardized differences compare controls to the transplant referent group. Significance is determined if the standardized difference between the two groups is $\geq 10 \% .{ }^{\dagger}$ Includes coronary artery bypass grafting (CABG) and percutaneous coronary intervention (PCI). ${ }^{\ddagger}$ Includes gastroscopy, colonoscopy, sigmoidoscopy, duodenoscopy, and esophagoscopy. Information on medications was limited to those $\geq 65$ years of age.

samples from the general population. The relative risk and the corresponding 95\% confidence intervals (CI) were calculated. For the general population comparison, the three-year incidence rate of the primary outcome in the transplant population was standardized based on age and sex distributions of the general population sample. For the matched analyses, individuals from the transplant and control groups were matched on a 1:1 ratio on age, sex, and logit of the propensity score. Covariates in our propensity model included year of cohort entry, rural location, income quartile, atrial fibrillation, stroke, major hemorrhage, myocardial infarction, deep vein thrombosis, pulmonary embolism, heart valve replacement, congestive heart failure, coronary artery disease, coronary revascularization, diabetes mellitus, hypertension, peripheral artery disease, and Charlson Comorbidity Index. Pairs were matched without replacement using greedy matching within a caliper width of 0.2 standard deviations (SD) of the logit of the propensity score. This model was further adjusted for hypertension to account for the imbalance on this covariate between the two groups at baseline after matching. Conditional logistic regression was used to obtain conditional odds ratios and $95 \%$ confidence intervals, accounting for the matched pairs. Given the low cumulative incidence, odds

Risk of Major Hemorrhage after Kidney Transplantation ratios were interpreted as relative risks. The three-year cumulative incidence of nontraumatic hemorrhage was further stratified by age group $(\leq 44,45-64, \geq 65$ years) and donor type (deceased or living donor). To examine the impact of postoperative hemorrhage risk, we determined the three-year cumulative incidence of nontraumatic major hemorrhage after excluding bleeding that occurred within first 90 days after transplantation.

\section{Results}

\section{Baseline Characteristics}

Our final cohort included 4,958 incident kidney transplant patients comprising 13,575 person-years of followup. The median follow-up was 3.0 years. Comparison of baseline characteristics between transplant recipients and a random sample of the general population is presented in table 1. Patients who received a transplant were older, 
Table 2. Additional baseline characteristics of kidney transplant recipients

\begin{tabular}{lc}
\hline Characteristics & $\begin{array}{c}\text { Total transplant } \\
\text { recipients }\end{array}$ \\
\hline & \\
$\mathrm{n}$ Race, \% & 4,958 \\
Caucasian & 68.33 \\
Asian & 6.47 \\
Black & 5.59 \\
Aboriginal & 1.47 \\
Other & 8.42 \\
Unknown & 9.72 \\
Cause of end-stage renal disease, \% & \\
Glomerulonephritis & 35.80 \\
Allergic interstitial nephritis & 2.04 \\
Obstruction & 4.50 \\
Polycystic kidney disease & 12.97 \\
Diabetes & 17.31 \\
Vascular disease & 9.98 \\
Other & 6.37 \\
Missing/unknown & 11.03 \\
Dialysis modality, \% & \\
Hemodialysis & 60.2 \\
Peritoneal dialysis & 2.9 \\
Missing & 30.2 \\
Vintage (median years) & 10.2 \\
Donor type, \% & 3.0 (IQR 1.0-4.5) \\
Living & \\
Deceased & 41.4 \\
Unknown/missing & 57.3 \\
Delayed graft function*, \% & 1.4 \\
Living & \\
Deceased & \\
Primary renal allograft non-function & \\
\hline & \\
\hline & \\
\hline
\end{tabular}

$\mathrm{IQR}=$ Interquartile range. ${ }^{*}$ Delayed graft function is defined as at least one dialysis code in the first 7 days post-transplant date. ${ }^{\dagger}$ Primary renal allograft non-function is defined as at least three codes for dialysis on separate days with at least one code occurring in the first 7 days, in 8-30 days, and in the 31-60 days post-transplant date.

more likely male but had similar neighborhood income compared to the general population. As expected, comorbidity was greater among kidney transplant recipients. Most transplant recipients were Caucasian and had received hemodialysis prior to transplantation (table 2). The median time of receiving dialysis prior to transplant was 3.0 years (interquartile range [IQR], 1.0 to 4.5 years). Over half the patients $(57.3 \%)$ received a transplant from a deceased donor (table 2). Delayed graft function occurred in roughly one-fifth of patients (18.7\%). Ninetysix percent $(4,756 / 4,958)$ of incident kidney transplant patients were age, sex, and propensity score matched to non-kidney transplant patients. The matched cohort baseline characteristics were similar except for hypertension (standardized difference 12\%) that was higher in the non-kidney transplant group (online suppl. table S4).

\section{Incidence of Hemorrhage}

Within the first three years of transplantation, 173 of 4,958 kidney transplant recipients were hospitalized with nontraumatic hemorrhage (3.5\%, 95\% CI 3.0-4.1\%) compared to 428 of 100,000 individuals in the general population $(0.4 \%, 95 \%$ CI $0.4-0.5 \%)$. The relative risk of hemorrhage was eight-fold higher in the kidney transplant recipients compared to the general population (RR 8.2; 95\% CI 6.9-9.7) (table 3). The corresponding crude rates of nontraumatic major hemorrhage were 12.7 per 1,000 person-years in transplant recipients versus 1.44 per 1,000 person-years in the general population. After standardizing age and sex to the general population, the standardized nontraumatic major hemorrhage rate of the transplant group was 11.4 events per 1,000 person-years. The age, sex, and propensity score matched transplant and general population groups had hemorrhage rates of 12.3 and 1.6 per 1,000 person years, respectively.

The three-year cumulative incidence of hemorrhage post-transplant and crude RR by the type of bleeding compared to the general population was as follows: lower gastrointestinal (LGI) (1.8\% vs. $0.2 \%$; RR 9.1, 95\% CI 7.111.7); upper gastrointestinal (UGI) (1.6\% vs. $0.2 \%$; RR 8.7, $95 \%$ CI 6.7-11.3); intra-cerebral (ICH) $(0.2 \%$ vs. 0.04\%; RR 6.2, 95\% CI 3.3-11.8); and subarachnoid (SAH) ( $\leq 0.1 \%$ vs. $0.03 \%$; RR $\leq 3.4,95 \%$ CI $1.3-8.7$ ) (table 3). In-hospital gastrointestinal-related endoscopic procedures were similarly increased among transplant recipients (7.9\% vs. $0.5 \%$; RR $15.6,95 \%$ CI $13.7-17.8)$ (table 3$)$. The three-year cumulative incidence for the age-, sex- and propensity score-matched transplant cohort and odds ratios compared to the transplant cohort were as follows: LGI 2.4\% (OR 1.7, 95\% CI 1.3-2.2); UGI 1.1\% (OR 1.6, 95\% CI 1.1-2.3); and ICH 0.2\% (OR 1.6, 95\% CI 0.64.1) (table 3).

\section{Subgroups Analyses}

There was a steady increase in the three-year cumulative incidence of major nontraumatic hemorrhage by age with an over two-fold increase between those $\leq 44$ and $\geq 65$ years (fig. 1). Similarly, the three-year cumulative incidence of nontraumatic hemorrhage was almost threefold higher in those who received a kidney from a deceased versus a living donor ( $4.8 \%$ vs. $1.8 \%)$. After exclu- 
Table 3. Three-year cumulative incidence and risk of non-traumatic major hemorrhage and related gastrointestinal endoscopy among kidney transplant recipients compared to a random sample from the general population, and compared to an age-, sex- and propensity score-matched cohort

\begin{tabular}{|c|c|c|c|c|c|c|}
\hline \multirow[t]{2}{*}{ Outcome } & \multicolumn{3}{|c|}{ Unmatched cohort } & \multicolumn{3}{|c|}{ Propensity score matched cohort } \\
\hline & $\begin{array}{l}\text { transplant } \\
(\mathrm{n}=4,958)\end{array}$ & $\begin{array}{l}\text { general } \\
\text { population } \\
(n=100,000)\end{array}$ & relative risk & $\begin{array}{l}\text { transplant } \\
(\mathrm{n}=4,756)\end{array}$ & $\begin{array}{l}\text { matched } \\
\text { population } \\
(\mathrm{n}=4,756)\end{array}$ & $\begin{array}{l}\text { adjusted } \\
\text { conditional } \\
\text { odds ratio }^{¥}\end{array}$ \\
\hline \multicolumn{7}{|l|}{ Total hemorrhage } \\
\hline $\mathrm{n}$ & 173 & 428 & & 165 & 114 & \\
\hline Cumulative incidence & $3.5(3.0-4.1)$ & $0.4(0.4-0.5)$ & $8.2(6.9-9.7)$ & $3.5(3.0-4.0)$ & $2.4(2.0-2.9)$ & $1.7(1.3-2.2)$ \\
\hline \multicolumn{7}{|c|}{ Upper gastrointestinal hemorrhage } \\
\hline $\mathrm{n}$ & 78 & 181 & & 74 & 51 & \\
\hline Cumulative incidence & $1.6(1.3-2.0)$ & $0.2(0.2-0.2)$ & $8.7(6.7-11.3)$ & $1.6(1.2-2.0)$ & $1.1(0.8-1.4)$ & $1.6(1.1-2.3)$ \\
\hline \multicolumn{7}{|c|}{ Lower gastrointestinal hemorrhage } \\
\hline $\mathrm{n}$ & 88 & 195 & & 84 & 58 & \\
\hline Cumulative incidence & $1.8(1.4-2.2)$ & $0.2(0.2-0.2)$ & $9.1(7.1-11.7)$ & $1.8(1.4-2.2)$ & $1.2(1.0-1.6)$ & $1.8(1.2-2.6)$ \\
\hline \multicolumn{7}{|l|}{ Intra-cerebral hemorrhage } \\
\hline $\mathrm{n}$ & 12 & 39 & & 9 & 11 & \\
\hline Cumulative incidence & $0.2(0.1-0.4)$ & $0.0(0.0-0.1)$ & $6.2(3.3-11.8)$ & $0.2(0.1-0.4)$ & $0.2(0.1-0.4)$ & $1.6(0.6-4.1)$ \\
\hline \multicolumn{7}{|l|}{ Sub-arachnoid hemorrhage } \\
\hline $\mathrm{n}$ & $\leq 5^{*}$ & 30 & & $\leq 5^{*}$ & $\leq 5^{*}$ & \\
\hline Cumulative incidence & $0.1(0.0-0.2)^{*}$ & $0.0(0.0-0.0)$ & $\leq 3.4(1.3-8.7)^{*}$ & $\leq 0.1(0.0-0.2)$ & $\leq 0.1(0.0-0.2)$ & $3.0(0.3-28.8)$ \\
\hline \multicolumn{7}{|l|}{ Other $^{\dagger}$} \\
\hline $\mathrm{n}$ & 11 & 18 & & 6 & 10 & \\
\hline Cumulative incidence & $0.2(0.1-0.4)$ & $0.0(0.0-0.0)$ & $12.3(5.8-26.1)$ & $0.1(0.1-0.3)$ & $0.2(0.1-0.4)$ & $1.8(0.6-4.9)$ \\
\hline \multicolumn{7}{|c|}{ Any gastrointestinal endoscopy } \\
\hline $\mathrm{n}$ & 389 & 503 & & 147 & 368 & \\
\hline Cumulative incidence & $7.9(7.1-8.6)$ & $0.5(0.5-0.6)$ & $15.6(13.7-17.8)$ & $3.1(2.6-3.6)$ & $7.7(7.0-8.5)$ & $2.9(2.3-3.6)$ \\
\hline
\end{tabular}

* Cell values were suppressed for the purposes of privacy and confidentiality. ${ }^{¥}$ Odds ratios were estimated using conditional logistic regression analysis and were further adjusted for hypertension. These values can be interpreted as relative risks in light of the low cumulative incidence in the exposure and control groups. ${ }^{\dagger}$ Other includes other nontraumatic intracranial hemorrhage. ${ }^{\ddagger}$ Includes gastroscopy, colonoscopy, sigmoidoscopy, duodenoscopy, and esophagoscopy.

sion of nontraumatic major hemorrhage events within 90 days of the transplantation procedure, the three-year cumulative incidence decreased from $3.5 \%$ to $2.3 \%$ (95\% CI 1.9-2.8\%). There was still a roughly five-fold increased risk of nontraumatic hemorrhage beyond 90 days posttransplantation compared to the general population. After excluding hemorrhages within the first 90 days posttransplant, the event rate decreased from 12.7 to 7.97 per 1,000 person years.

\section{Discussion}

In this large retrospective cohort study, we identified a considerable risk for major nontraumatic hemorrhage among kidney transplantation recipients and this was significantly higher than in the general population. The three-year risk was greater among kidney transplant re-

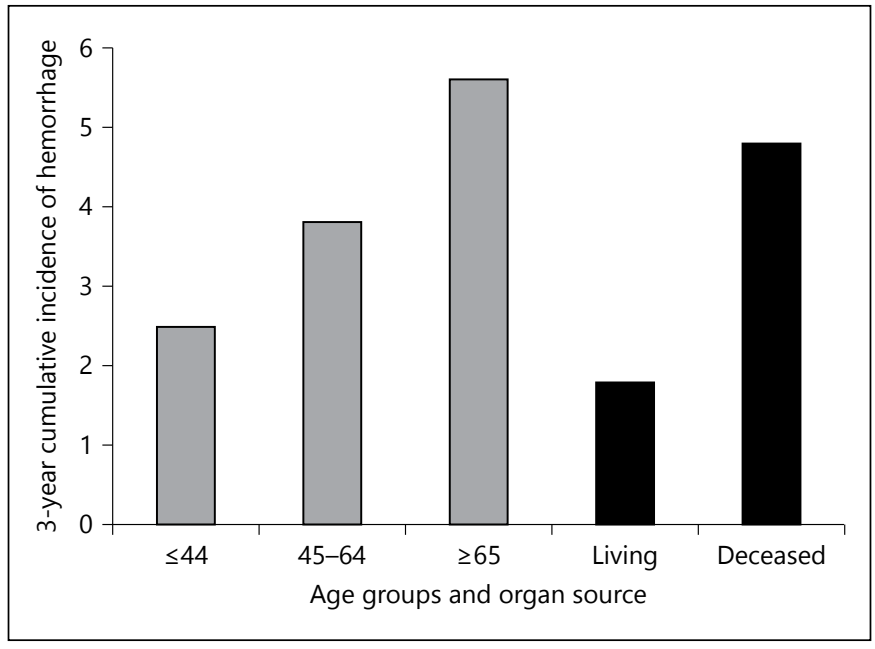

Fig. 1. The three-year cumulative incidence of nontraumatic major hemorrhage in kidney transplant recipients by recipient age group at the time of transplantation and kidney donor type. 
cipients compared to the general population across all subtypes of hemorrhages and for related in-hospital gastrointestinal endoscopic procedures. The increased risk of hemorrhage was attenuated but was still statistically significant after matching on age, sex, and propensity score. There was more than a two-fold increase in the incidence of hemorrhage among the oldest age group compared to the youngest and among recipients of a kidney from a deceased versus living donor. Approximately, two-thirds of events occurred after the immediate postoperative period ( $>90$ days). Taken together, this study highlights the burden of hemorrhage in kidney transplant recipients and the need to develop and evaluate preventative strategies.

We are the first, to our knowledge, to report the incidence of total hemorrhage in the kidney transplantation population. Previous reports are limited to single case reports or case series, medication- or cause-specific hemorrhagic complications, or peri-/postoperative-related hemorrhage [6, 9-12, 27-30]. In contrast, our study provides a cumulative picture of the impact of hemorrhage and its associated hospitalizations in kidney transplant recipients.

We examined the specific subtypes of gastrointestinal and central nervous system-related hemorrhage and found 2- to 9-fold increases in incidence of each subtype relative to the general population. There are few, and often indirect, previous studies available for comparison [31-34]. The increase in hemorrhage risk is not surprising as in general, risk factors for specific subtypes of hemorrhage appear to be more common among kidney transplantation recipients. Upper and lower gastrointestinal lesions such as duodenal ulcers, gastritis, $H$. pylori infection, and solid tumors are more common among kidney transplant recipients [33, 34]. An American database (USRDS) study reported a considerably higher threeyear cumulative incidence of intra-cerebral hemorrhage of $1.1 \%$ (95\% CI $1.0-1.2 \%$ ) compared to the $0.2 \%$ (95\% CI $0.1-0.4 \%)$ observed in our cohort [32]. This discrepancy may be due to the higher proportion of African Americans and earlier study era in the American study. Of note, intracerebral hemorrhage was associated with a particularly poor outcome with an adjusted hazard ratio of 10.6 (95\% CI 8.9-12.5) for mortality illustrating the specific importance and poor outcomes associated with hemorrhage in this site [32].

The elevated risk of hemorrhage is consistent with, but lower, than the risk in the dialysis population. Using a similar definition, we previously reported a three-year incidence of hemorrhage of $14.4 \%$ (four-fold elevation) among greater than 11,000 dialysis patients compared to the general population [35]. Reasons for the differing risk between the two populations include selecting a healthier segment of the dialysis population for transplantation, medication exposures (for example, heparin during intermittent hemodialysis), uremia-related platelet dysfunction, and vascular access complications [36, 37]. It appears the receipt of a kidney transplant may attenuate some of the hemorrhage risk. Lentine et al. compared transplant recipients to individuals on the waiting list, reporting a reduction in the hemorrhagic stroke risk following transplantation [32]. Whether kidney transplantation in and of itself reduces the risk of other subtypes of hemorrhage remains unknown.

The risk of bleeding was not uniform and increased with age and when the organ was obtained from a deceased rather than a living donor and was highest in the first 90 days post-transplantation. Age is a well-recognized and reported risk factor for hemorrhage in the general population often representing the accrual of comorbid illnesses, medications, and hemorrhage-related pathology $[2,4,5]$. Receipt of a decreased donor organ is associated with an increased risk of complications such as delayed graft function, need for early renal replacement therapy, and escalation of immunosuppressive therapies all of which may contribute to the hemorrhage risk [38]. Similarly, one-third of hemorrhages occurred in the first three months after the transplantation procedure, the period when procedure-related complications, maximal doses of steroids and immunosuppressive and the need for renal replacement therapy are highest. Despite this, the majority of hemorrhagic events occurred after the initial three-month period, indicating there are also considerable longer-term risks.

Our study quantifies the significant burden of hemorrhage in the kidney transplant population highlighting the need for monitoring and population-specific interventions to reduce the risk. Advancement in surgical techniques, elimination of the use of anticoagulants in the peri- and immediate post-surgical period and minimization of steroid-use represent interventions that could reduce the hemorrhage risk in the early post-transplant period $[11,29,39,40]$. Evidence for monitoring and interventions to reduce the longer-term risk of hemorrhage is limited. Aggressive cancer screening, $H$. pylori eradication, careful consideration of the risks/benefits of antithrombotic medications, and the role of acid suppressing medications represent areas for further investigation. Use of in-hospital, gastrointestinal-related endoscopies for screening and/or therapeutic interventions around the
Sood et al. 
time of hemorrhagic events occurred commonly in our cohort at $7.85 \%$ (15-fold increase compared to the general population). However, the role and optimal periodicity of endoscopic outpatient screening remains unclear. Other screening strategies such as fecal occult blood testing for colonic lesions and neurological imaging for aneurysms in specific populations (polycystic kidney disease) are supported by evidence and should be utilized $[33,41,42]$.

There are limitations to our study that warrant mention. We considered only nontraumatic major hemorrhage validated by previous studies and not minor hemorrhages such as hemoptysis or epistaxis as these are currently not validated. We did not include recurrent events of the same sub-type of hemorrhage so our findings may represent an underestimate of the true burden of hemorrhage in this population. We examined only hemorrhagic and endoscopic events at hospitalization and thus did not include hemorrhages or procedures from the outpatient setting. We did not account for medication usage that may contribute to the hemorrhage risk, including warfarin or anti-platelet usage, given that only a subset of our recipients would be covered by our provincial drug plan with data captured by our administrative databases. In our adjusted analyses we accounted for co-morbid conditions where antithrombotic medication prescription is common (example coronary artery disease) and this could partially reduce confounding by these medications. Transplantation patients may undergo more intensive monitoring and follow-up resulting in better capture of events. We also lacked measures of graft function at the time of hemorrhage. Lastly, although we used a matched propensity score analysis, there remains the possibility of unmeasured confounders.

In conclusion, we found a significant increase in the risk of nontraumatic major hemorrhage among kidney transplant recipients compared to the general population. The risk of hemorrhage increases with age, receipt of a deceased donor kidney and is highest in the early post- transplantation period. Further investigations of screening and targeted interventions to reduce the burden of hemorrhage in this population are warranted.

\section{Acknowledgments}

This study was conducted at the Institute for Clinical Evaluative Sciences (ICES) Western Site. ICES is funded by an annual grant from the Ontario Ministry of Health and Long-Term Care. ICES Western is funded by an operating grant from the Academic Medical Organization of Southwestern Ontario. This project was conducted with members of the provincial ICES Kidney, Dialysis and Transplantation Research Program (www.ices.on.ca), which receives programmatic grant funding from the Canadian Institutes of Health Research. MMS receives a salary support award from the Jindal research chair for the prevention of kidney disease. GAK receives a salary support award from the University of Ottawa Chair in Clinical Transplantation Research. NNL and AM were supported by a Kidney Research Scientist Core Education and $\mathrm{Na}$ tional Training Program (KRESCENT) post-doctoral fellowship award. NNL was supported by the Clinical Investigator Program at Western University. $\mathrm{KN}$ is supported by the Canadian Institutes of Health Research Allied Health Professional Fellowship. MKK is supported by a Career Investigator award from the Heart and Stroke Foundation, Ontario provincial office.

\section{Role of the Sponsor}

The opinions, results and conclusions reported in this paper are those of the authors and are independent of the funding sources.

\section{Disclosure Statement}

None declared.

\section{Contributions}

M.M.S., A.X.G. and G.K. conceptualized the study. M.M.S. drafted the first copy of the manuscript. E.M. completed the data analysis. S.E.B., L.M., K.N., M.K., S.J.K., N.L., A.M., Z.V., J.P. all contributed to the data interpretation and manuscript.

\section{References}

1 Ohmann C, Imhof M, Ruppert C, Janzik U, Vogt C, Frieling T, et al: Time-trends in the epidemiology of peptic ulcer bleeding. Scand J Gastroenterol 2005;40:914-920.

$\checkmark 2$ van Leerdam ME: Epidemiology of acute upper gastrointestinal bleeding. Best Pract Res Clin Gastroenterol 2008;22:209-224.

-3 Laine L, Yang H, Chang SC, Datto C: Trends for incidence of hospitalization and death due to GI complications in the United States from 2001 to 2009. Am J Gastroenterol 2012;107: 1190-1195.

4 Zuccaro G: Epidemiology of lower gastrointestinal bleeding. Best Pract Res Clin Gastroenterol 2008;22:225-232.

-5 Østbye T, Levy AR, Mayo NE: Hospitalization and case-fatality rates for subarachnoid hemorrhage in Canada from 1982 through 1991. The
Canadian Collaborative Study Group of Stroke Hospitalizations. Stroke 1997;28:793-798.

-6 Troppmann C, Papalois BE, Chiou A, Benedetti E, Dunn DL, Matas AJ, Najarian JS, Gruessner RW: Incidence, complications, treatment, and outcome of ulcers of the upper gastrointestinal tract after renal transplantation during the cyclosporine era. J Am Coll Surg 1995;180:433-443. 
7 Wijdicks EF, Torres VE, Schievink WI, Sterioff S: Cerebral hemorrhage in recipients of renal transplantation. Mayo Clin Proc 1999;74: 1111-1112.

8 Baumert H: Fatal and nonfatal hemorrhagic complications of living kidney donation. Ann Surg 2006;244:629.

9 Vincent F, Bensousan TA: Re: Nitrofurantoin-induced pulmonary hemorrhage in a renal transplant recipient receiving immunosuppressive therapy: case report and review of the literature. J Urol 1995;154:214.

10 Vlahakis NE, Rickman OB, Morgenthaler T: Sirolimus-associated diffuse alveolar hemorrhage. Mayo Clin Proc 2004;79:541-545.

11 Kusyk T, Verran D, Stewart G, Ryan B, Fisher $\mathrm{J}$, Tsacalos K, et al: Increased risk of hemorrhagic complications in renal allograft recipients receiving systemic heparin early posttransplantation. Transplant Proc 2005;37: 1026-1028.

12 Osman Y, Kamal M, Soliman S, Sheashaa H, Shokeir A, Shehab El-Dein AB: Necessity of routine postoperative heparinization in nonrisky live-donor renal transplantation: results of a prospective randomized trial. Urology 2007;69:647-651.

13 Hernández-Díaz S, Rodríguez LA: Incidence of serious upper gastrointestinal bleeding/ perforation in the general population: review of epidemiologic studies. J Clin Epidemiol 2002;55:157-163.

14 Stats Canada: Population by year, by province and territory (number). www.76.statcan.gc. ca. Statistics Canada, 2012.

-15 von Elm E, Altman DG, Egger M, Pocock SJ, Gøtzsche PC, Vandenbroucke JP: The Strengthening the Reporting of Observational Studies in Epidemiology (STROBE) statement: guidelines for reporting observational studies. Ann Intern Med 2007;147:573-577.

16 Canadian Institute for Health Information: Treatment of End-Stage Organ Failure in Canada, 1999-2008: CORR 2010 Annual Report. Ottawa, Ontario, CIHI, 2010.

17 Garg AX, Pouget J, Young A, Huang A, Boudville N, Hodsman A, et al: Fracture risk in living kidney donors: a matched cohort study. Am J Kidney Dis 2012;59:770-776.

18 Thomas SM, Lam NN, Welk BK, Nguan C, Huang A, Nash DM, et al: Risk of kidney stones with surgical intervention in living kidney donors. Am J Transplant 2013;13:29352944.

19 Garg AX, Meirambayeva A, Huang A, Kim J, Prasad GV, Knoll G, et al: Cardiovascular disease in kidney donors: matched cohort study. BMJ 2012;344:e1203.
20 Gilbert CJ, Gomes T, Mamdani MM, Hellings C, Yao Z, Garg AX, et al: No increase in adverse events during aliskiren use among ontario patients receiving angiotensin-converting enzyme inhibitors or angiotensin-receptor blockers. Can J Cardiol 2013;29:586-591.

-21 Siddiqui NF, Coca SG, Devereaux PJ, Jain AK, Li L, Luo J, et al: Secular trends in acute dialysis after elective major surgery - 1995 to 2009. CMAJ 2012;184:1237-1245.

22 Young A, Kim SJ, Speechley MR, Huang A, Knoll GA, Prasad GV, et al: Accepting kidneys from older living donors: impact on transplant recipient outcomes. Am J Transplant 2011;11:743-750.

23 Pisters R, Lane DA, Nieuwlaat R, de Vos CB, Crijns HJ, Lip GY: A novel user-friendly score (HAS-BLED) to assess 1-year risk of major bleeding in patients with atrial fibrillation: the Euro Heart Survey. Chest 2010;138:10931100 .

24 Kokotailo RA, Hill MD: Coding of stroke and stroke risk factors using international classification of diseases, revisions 9 and 10. Stroke 2005;36:1776-1781.

25 Arnason T, Wells PS, van Walraven C, Forster AJ: Accuracy of coding for possible warfarin complications in hospital discharge abstracts. Thromb Res 2006;118:253-262.

26 Iribarren C, Jacobs DR, Sadler M, Claxton AJ, Sidney S: Low total serum cholesterol and intracerebral hemorrhagic stroke: is the association confined to elderly men? The Kaiser Permanente Medical Care Program. Stroke 1996; 27:1993-1998.

27 Blaschke S, Grunewald W, Strutz F, Sattler B Müller GA, Reuss-Borst M: Fulminant alveolar haemorrhage in a case of recurrent small vessel vasculitis after renal transplantation. Rheumatology (Oxford) 2000;39:10421044

28 Champion L, Stern M, Israël-Biet D, MamzerBruneel MF, Peraldi MN, Kreis H, et al: Brief communication: sirolimus-associated pneumonitis: 24 cases in renal transplant recipients. Ann Intern Med 2006; 144:505-509.

29 Mathis AS, Davé N, Shah NK, Friedman GS: Bleeding and thrombosis in high-risk renal transplantation candidates using heparin. Ann Pharmacother 2004;38:537-543.

30 Sachdeva A, Matuschak GM: DIffuse alveolar hemorrhage following alemtuzumab. Chest 2008;133:1476-1478.

-31 Oliveras A, Roquer J, Puig JM, Rodríguez A, Mir M, Orfila MA, et al: Stroke in renal transplant recipients: epidemiology, predictive risk factors and outcome. Clin Transplant 2003; $17: 1-8$
32 Lentine KL, Rocca Rey LA, Kolli S, Bacchi G, Schnitzler MA, Abbott KC, et al: Variations in the risk for cerebrovascular events after kidney transplant compared with experience on the waiting list and after graft failure. Clin J Am Soc Nephrol 2008;3:1090-1101.

33 Collins MG, Teo E, Cole SR, Chan CY, McDonald SP, Russ GR, et al: Screening for colorectal cancer and advanced colorectal neoplasia in kidney transplant recipients: cross sectional prevalence and diagnostic accuracy study of faecal immunochemical testing for haemoglobin and colonoscopy. BMJ 2012;345:e4657.

34 Khedmat $\mathrm{H}$, Ahmadzad-Asl M, Amini M, Lessan-Pezeshki M, Einollahi B, Pourfarziani $\mathrm{V}$, et al: Gastro-duodenal lesions and helicobacter pylori infection in uremic patients and renal transplant recipients. Transplant Proc 2007;39:1003-1007.

35 Sood MM, Bota SE, McArthur E, Kapral MK, Tangri N, Knoll G, Zimmerman D, Garg AX: The three-year incidence of major hemorrhage among older adults initiating chronic dialysis. Canadian J Kidney Health Dis 2014. $1: 21$.

36 Holden RM, Harman GJ, Wang M, Holland D, Day AG: Major bleeding in hemodialysis patients. Clin J Am Soc Nephrol 2008;3:105110

37 Sood MM, Larkina M, Thumma JR, Tentori F, Gillespie BW, Fukuhara S, et al: Major bleeding events and risk stratification of antithrombotic agents in hemodialysis: results from the DOPPS. Kidney Int 2013;84:600-608.

- 38 Yarlagadda SG, Coca SG, Formica RN Jr, Poggio ED, Parikh CR: Association between delayed graft function and allograft and patient survival: a systematic review and meta-analysis. Nephrol Dial Transplant 2009;24:10391047.

39 Pascual J, Galeano C, Royuela A, Zamora J: A systematic review on steroid withdrawal between 3 and 6 months after kidney transplantation. Transplantation 2010;90:343-349.

40 Osman Y, Shokeir A, Ali-el-Dein B, Tantawy M, Wafa EW, el-Dein AB, et al: Vascular complications after live donor renal transplantation: study of risk factors and effects on graft and patient survival. J Urol 2003;169:859-862.

41 Grantham JJ: Clinical practice. Autosomal dominant polycystic kidney disease. N Engl J Med 2008;359:1477-1485.

42 Knoll G, Cockfield S, Blydt-Hansen T, Baran D, Kiberd B, Landsberg D, et al: Canadian society of transplantation: consensus guidelines on eligibility for kidney transplantation. CMAJ 2005; 173:S1-S25. 